\title{
Correlation between monoamine neurotransmitter and cytokine levels and the occurrence of post-traumatic stress disorder among operating room nurses
}

\author{
Yajuan Ke ${ }^{1}$, Hongying Zhu ${ }^{1}$, Yi Huang ${ }^{1}$, Liurong $\mathrm{Cheng}^{1}$, Xiaofang $\mathrm{Wu}^{2}$, Nanyao Chen ${ }^{3}$ \\ ${ }^{1}$ Department of Operating Room, Hainan General Hospital, Haikou, China; ${ }^{2}$ Department of Operating Room, the Second Affiliated Hospital of \\ Hainan Medical University, Haikou, China; ${ }^{3}$ Department of Operating Theater, The Fourth People's Hospital of Haikou, Haikou, China \\ Contributions: (I) Conception and design: Y Ke; (II) Administrative support: Y Ke, H Zhu; (III) Provision of study materials or patients: Y Ke, Y \\ Huang; (IV) Collection and assembly of data: H Zhu, L Cheng; (V) Data analysis and interpretation: X Wu, N Chen; (VI) Manuscript writing: All \\ authors; (VII) Final approval of manuscript: All authors. \\ Correspondence to: Yi Huang. Operating Room, Hainan People's Hospital, No. 19 Xiuhua Road, Haikou, China. Email: huangyi201808@163.com.
}

\begin{abstract}
Background: The aim of the present study was to investigate the correlation between monoamine neurotransmitter and cytokine levels and the occurrence of post-traumatic stress disorder (PTSD) among operating room nurses.
\end{abstract}

Methods: A total of 131 nursing staff were selected and assigned into the PTSD, non-PTSD, and control group. Enzyme-linked immunosorbent assay was applied to determine the monoamine neurotransmitters in plasma and serum cytokines. Receiver-operating characteristic curve analysis was conducted to assess the sensitivity and specificity of neurotransmitters and cytokines in the clinical detection of PTSD among operating room nurses. Addenbrooke's Cognitive Examination-Revised and the Connor-Davidson Resilience Scale were used to evaluate the correlation between neurotransmitter and cytokine levels and the clinical characteristics of operating room nurses with PTSD.

Results: Our study found that the monoamine neurotransmitters and cytokines among nurses in the PTSD group were significantly higher than those in the non-PTSD and control group. Neurotransmitter and cytokine levels as clinical predictors of PTSD among operating room nurses have good sensitivity and specificity, and were negatively correlated with cognitive function and resilience.

Conclusions: The findings of the present study confirm that monoamine neurotransmitter and cytokine levels are correlated with the occurrence of PTSD among operating room nurses.

Keywords: Post-traumatic stress disorder (PTSD); monoamine neurotransmitters; cytokines; operating room nurses; Addenbrooke's Cognitive Examination-Revised; Connor-Davidson Resilience Scale

Submitted Aug 25, 2020. Accepted for publication Nov 17, 2020.

doi: 10.21037/apm-20-1829

View this article at: http://dx.doi.org/10.21037/apm-20-1829

\section{Introduction}

Post-traumatic stress disorder (PTSD) occurs following exposure to a traumatic event, and is defined by obvious symptoms of re-experience, avoidance, and numbness persisting for $>1$ month after trauma (1). PTSD has been reported to be a serious and even disabling condition that affects about $8 \%$ of people at some point during their life (2). Statistics have demonstrated that PTSD is more common among women, and the morbidity rate has been found to be associated with trauma exposure rates (3). The cause of PTSD is well known; events involving threats to the physical integrity of oneself or others, and reactions to strong fear, horror, or helplessness (4). Patients with PTSD not only present intense negative emotional reactions when reminded of their trauma, but also show exaggerated anhedonia, social withdrawal, and emotional 
numbing (5). Patients with PTSD are more likely to have major depression, substance dependence, as well as lost the drive to live (6). Psychotherapy has been reported to be the mainstay of PTSD treatment (7). Psychological interventions for PTSD are not always effective and can leave some individuals with enduring symptoms. Prolonged exposure is an effective evidenced-based treatment for PTSD, but it is limited to certain population. However, the mechanisms underlying PTSD symptoms are not yet fully understood.

Neurotransmitters play a crucial role in maintaining physiological function at both the cellular and organ levels, and their imbalances have demonstrated a correlation in the pathology of several diseases (8). Monoamine neurotransmitters, a category of neurotransmitters, can act as biomarkers for the diagnosis and prognosis of some neurological disorders, such as Parkinson's disease and Alzheimer's disease $(9,10)$. It has also been shown that changes in neurotransmitter levels during early development can result in serious neurologic problems (11). Previously published studies have found immune alterations in PTSD patients, including increased levels of inflammatory cytokines (12). Former findings suggest that a generalized inflammatory state may be present in individuals with PTSD. PTSD is associated with heightened expression of several pro-inflammatory cytokines. The incidence of PTSD and its emotional associations among frontline health and rescue workers have been widely explored recently. Nurses are considered to be an increased risk of work-related stress, particularly those working in specialty areas, such as the intensive care unit, emergency room, as well as oncology departments (13). High PTSD rates have been reported among critical care nurses and pediatric nurses $(14,15)$. In the present study, we aimed to investigate the relationship between monoamine neurotransmitter and cytokine levels with the occurrence of PTSD among operating room nurses.

We present the following article in accordance with the MDAR reporting checklist (available at http://dx.doi. org/10.21037/apm-20-1829).

\section{Methods}

\section{Ethics statement}

The present study was approved by the Medical Ethics Committee of the Hainan General Hospital (No.:
20191023), and the participants provided signed informed consent. The study was conducted in accordance with the Declaration of Helsinki (as revised in 2013).

\section{Study participants}

The diagnostic criteria of PTSD (PTSD Checklist-Civilian Version) in the Diagnostic and Statistical Manual of Mental Disorders 4th edition was used to evaluate participants who experienced trauma 1 month after the traumatic event, or participants who did not experience trauma within 7 days of enrolment (16). The evaluation was carried out by psychology professionals, and scores ranged from 17 to 85 points. According to the international score and diagnostic criteria, a score of $\leq 37$ indicated no PTSD; 38-49 indicated a certain degree of PTSD symptoms, but not enough to be diagnosed as PTSD; and $\geq 50$ indicated PTSD, as diagnosed by two psychiatrists.

Between January 2019 and July 2019, 131 nursing staff were selected from the operating room at Hainan Provincial People's Hospital, the Second affiliated Hospital of Hainan Medical College, and other cooperative units. Of these, 45 nurses were diagnosed as having PTSD (PTSD group), 46 nurses had a certain degree of PTSD symptoms, but not enough to be diagnosed as PTSD (non-PTSD group), and 40 nurses were diagnosed as having no PTSD symptoms (control group). The team members learned about the nurses in the operating room to ensure that they had not experienced any new major psychological and physical traumas in the past month.

\section{Determination of plasma monoamine neurotransmitters and serum cytokines}

Participants were asked to not take any medications 3 days before testing. Elbow vein blood $(5 \mathrm{~mL})$ was taken on an empty stomach from 6:30 to 7:00 a.m; $2 \mathrm{~mL}$ of this was used to determine monoamine neurotransmitters in plasma, and the level of cytokines was measured in the upper serum after centrifugation of the residual $3 \mathrm{~mL}$. The enzymelinked adsorption reaction kit provided by Shanghai West Tang Biotechnology (China) was used to determine the monoamine neurotransmitters (norepinephrine and dopamine) in plasma and serum cytokines [interleukin (IL)2 , IL-6, IL-10, and tumor necrosis factor- $\alpha$ (TNF- $\alpha$ )] by an avidin-biotin complex enzyme-linked immunosorbent assay. Each sample were measured for three times. 


\section{Addenbrooke's Cognitive Examination-Revised (ACE-R)}

The ACE-R score is used to assess cognitive ability (17). It contains 5 subtests, each representing a cognitive domain: attention/orientation (18 points), memory (26 points), fluency (14 points), language (26 points), and visuospatial (16 points). The maximum score of the ACE-R is 100 , and the score of the average person is $>86$.

\section{Connor-Davidson Resilience Scale (CD-RISC)}

The 10-item CD-RISC (CD-RISC-10) is used to assess resilience (18). The CD-RISC-10 (a simple version of the CD-RISC) is used to assess PTSD severity and resilience. The 10 items were scored on a 5 -point scale with a full score of 40; the higher the score, the greater the resilience.

\section{Data analysis}

All data analyses were conducted using SPSS version 21.0 software (IBM, USA). All data were consistent with normal distribution by Kolmogorov-Smirnov test, and expressed as mean \pm standard deviation. Comparison of data between two groups was conducted by paired $t$-test, whereas comparison among multiple groups was conducted by 1-way analysis of variance. Enumeration data was analyzed by Fisher's test, and the correlation analysis was verified by Pearson's correlation coefficient test. A receiver-operating characteristic (ROC) curve was drawn to estimate the predictive efficacy of plasma monoamine neurotransmitters and serum cytokines on the occurrence of PTSD among operating room nurses. $\mathrm{P}<0.05$ was considered statistically significant.

\section{Results}

\section{Demographic characteristics}

A total of 131 operating room nursing staff were included in the present study (15 males and 116 females). Of these, 59 were aged $<30$ years, and 72 were aged $\geq 30-50$ years. Participants' demographic characteristics are shown in Table 1. After correction of the clinical data, no significant difference in demographic characteristics was found between the three groups $(\mathrm{P}>0.05)$.

\section{Plasma monoamine neurotransmitter and serum cytokine levels among operating room nurses}

The ELISA kit was used to detect norepinephrine, dopamine, IL-2, IL-6, IL-10, and TNF- $\alpha$ levels among nurses. The results showed that nurses in the control group showed the lowest levels of norepinephrine, dopamine, IL-2, IL-6, IL-10, and TNF- $\alpha$. However, monoamine neurotransmitter and cytokine levels among nurses in the non-PTSD group differed to those in the control group, but the difference was not significant. The monoamine neurotransmitter and cytokine levels of nurses in the PTSD group were significantly higher than those in the non-PTSD and control groups (Figure 1). The findings suggest that plasma monoamine neurotransmitter and serum cytokine levels are related to the occurrence of PTSD among operating room nurses. PTSD can lead to inflammatory reactions in vivo, and PTSD can increase neurotransmitter levels, which may lead to abnormal reaction of nervous system.

\section{Predictive efficacy of plasma monoamine neurotransmitters and serum cytokines on the occurrence of PTSD among operating room nurses}

To further evaluate the predictive efficacy of plasma monoamine neurotransmitter and serum cytokine levels on PTSD among operating room nurses, we analyzed 91 operating room nurses, who experienced psychotraumatic events, by ROC curve. The findings suggested that plasma monoamine neurotransmitter and serum cytokine levels had a good diagnostic effect on the occurrence of PTSD among operating room nurses, and could be used to predict PTSD (Figure 2).

\section{Relationship between plasma monoamine neurotransmitter and serum cytokine levels with cognitive behavior among operating room nurses with PTSD}

Cognition is a process of acquiring, understanding, and retaining knowledge; it involves a series of casual, psychological, and social behaviors, such as learning, memory, language, thinking, and emotion. It has been reported that PTSD patients have different degrees of cognitive impairment (19). Therefore, we applied the ACE-R to assess the cognitive level of nurses with PTSD. The ACE-R takes between 12 and 20 min (average, $16 \mathrm{~min}$ ) to administer and score in the clinical setting. The findings indicated that operating room nurses with PTSD had an average ACE-R score of $74.8 \pm 5.4$, which was negatively correlated with monoamine neurotransmitters and cytokines (Figure 3). 
Table 1 Comparison of demographic characteristics of the enrolled patients in the three groups

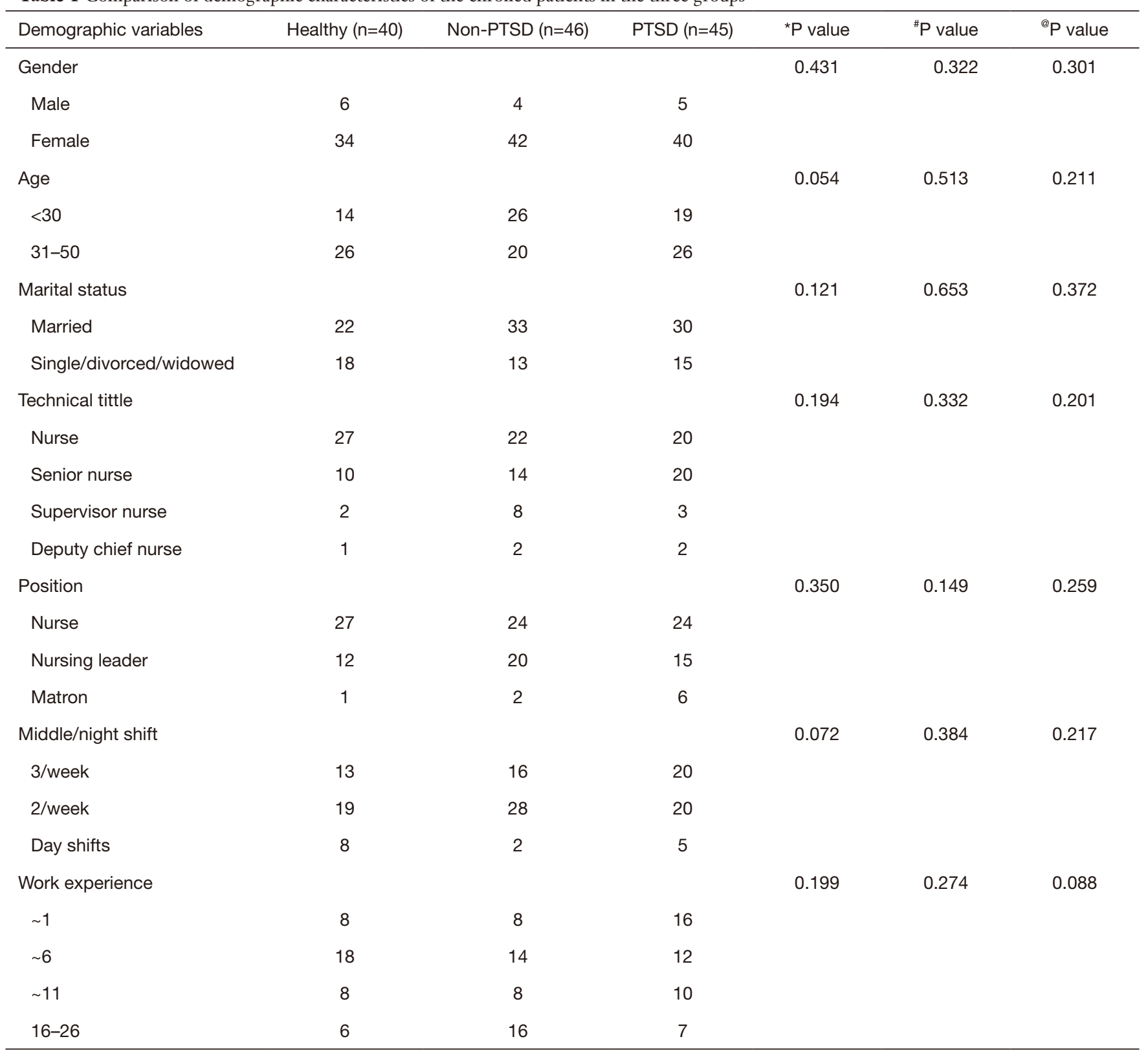

*, represented healthy group vs. non-PTSD group; ${ }^{\text {, }}$, represented healthy group vs. PTSD group; ${ }^{\circledR}$, represented PTSD group vs. non-PTSD group. Chi-square test was utilized to determine statistical significance. For healthy group, $\mathrm{N}=40$, while in non-PTSD group and PTSD group, $\mathrm{N}=46$ and 45 , respectively. PTSD, post-traumatic stress disorder.

Relationship between plasma monoamine neurotransmitter and serum cytokine levels with resilience among operating room nurses with PTSD

Resilience refers to the body's response to the changing environment. State is a dynamic form with a certain range of variation that changes with the environment, and achieves dynamic regulation and adaptation of the environment in the change. The score of the CD-RISC-10 ranges from 0 to 40 . The lower the score, the lower the resilience and the greater the PTSD severity. Therefore, the relationship between the plasma monoamine neurotransmitter and serum cytokine levels with the degree of PTSD can be further determined by analyzing these levels and resilience 
A
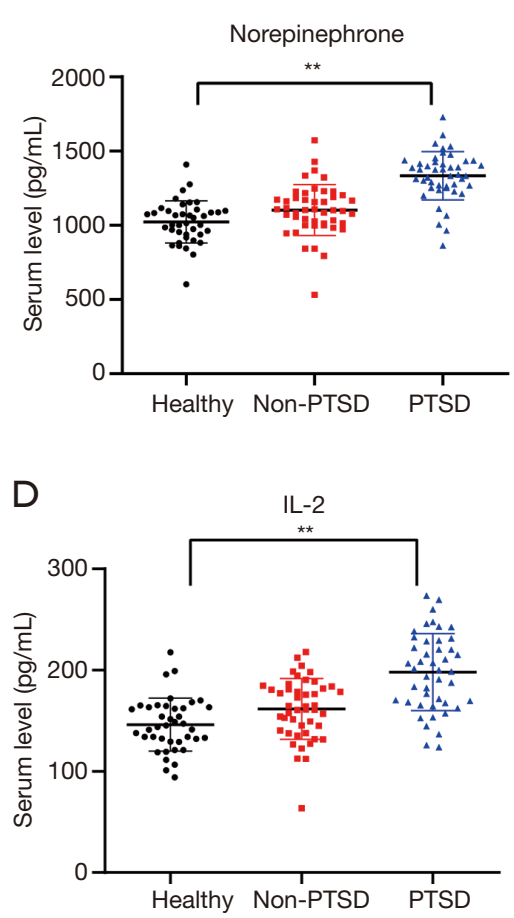

B

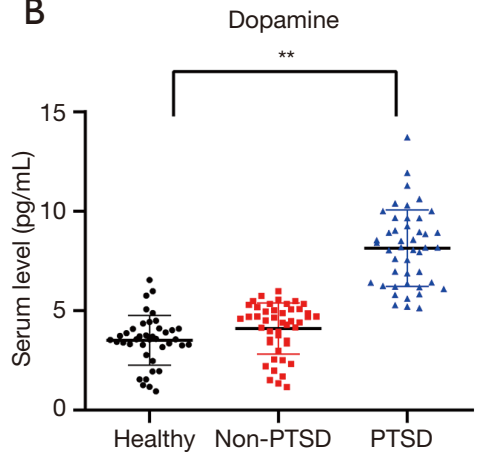

E

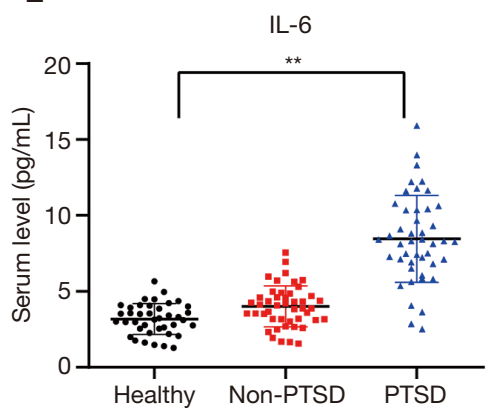

C

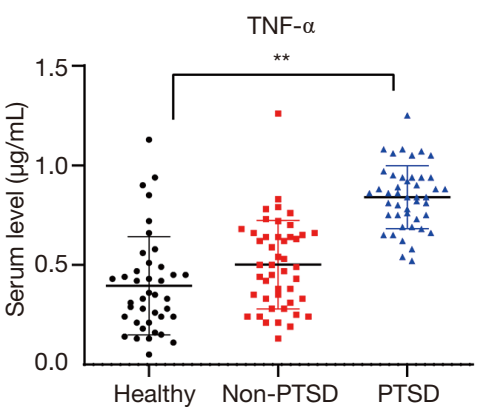

F

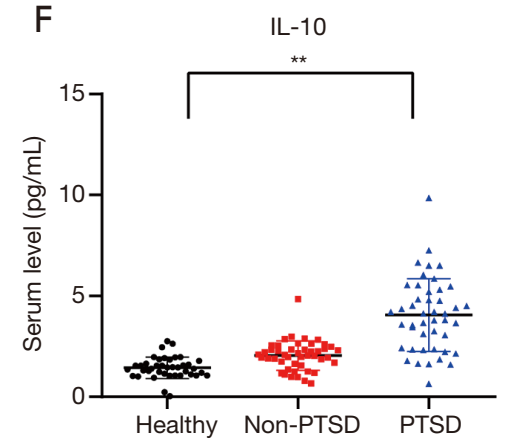

Figure 1 Monoamine neurotransmitter and cytokine levels among operating room nurses in the three groups. One-way analysis of variance was used to determine statistical significance. Data are expressed as mean \pm standard deviation. Control group: n=40; non-PTSD group: n=46; PTSD group: n=45. (A) Norepinephrine levels of all groups; (B) Dopamine levels of all groups; (C) TNF- $\alpha$ levels of all groups; (D) IL-6 levels of all groups; (E) IL-2 levels of all groups; (F) IL-10 levels of all groups. ${ }^{* *}, \mathrm{P}<0.05$; PTSD, post-traumatic stress disorder.

among operating room nurses with PTSD. The findings indicated that there was negative correlation between plasma monoamine neurotransmitter and serum cytokine levels with resilience. It is suggested that the higher the neurotransmitter and cytokine levels, the greater the PTSD severity (Figure 4).

\section{Discussion}

PTSD is a psychological disorder that develops in some people who have experienced traumatic or life-threatening events (20). PTSD can lead to decreased physical recovery, social functioning, and quality of life, and a higher risk of suicide attempts. A previously published study found that nurses are an increased risk of work-related stress, and are likely to suffer from PTSD (13). The aim of the present study was to investigate the correlation between monoamine neurotransmitter and cytokine levels and the occurrence of PTSD among operating room nurses.

First, we analyzed plasma monoamine neurotransmitter levels among operating room nurses, and found that these were related to the occurrence of PTSD. The biologic dysregulation of PTSD could be seen in physiological, neurohormonal, immunologic, as well as functional neuroanatomical levels (21). Early studies have suggested that increased noradrenaline activity may lead to impairment of medial prefrontal cortex function and a decrease of fear, which could explain the increase in behavioral indicators of anxiety and severity in PTSD (22-24). In line with the findings in the present study, a previously published study demonstrated that PTSD patients showed higher plasma concentrations of noradrenaline, adrenaline, and dopamine compared with controls (24).

A recent study reveals that PTSD is associated with heightened expression of several individual cytokines including IL-2, IL-4, IL-6, IFN- $\gamma$, and TNF- $\alpha$. In addition, serum cytokine levels among operating room nurses were also detected, and found that these levels were related to the occurrence of PTSD. PTSD could lead to inflammatory reactions in vivo and could increase neurotransmitter levels 

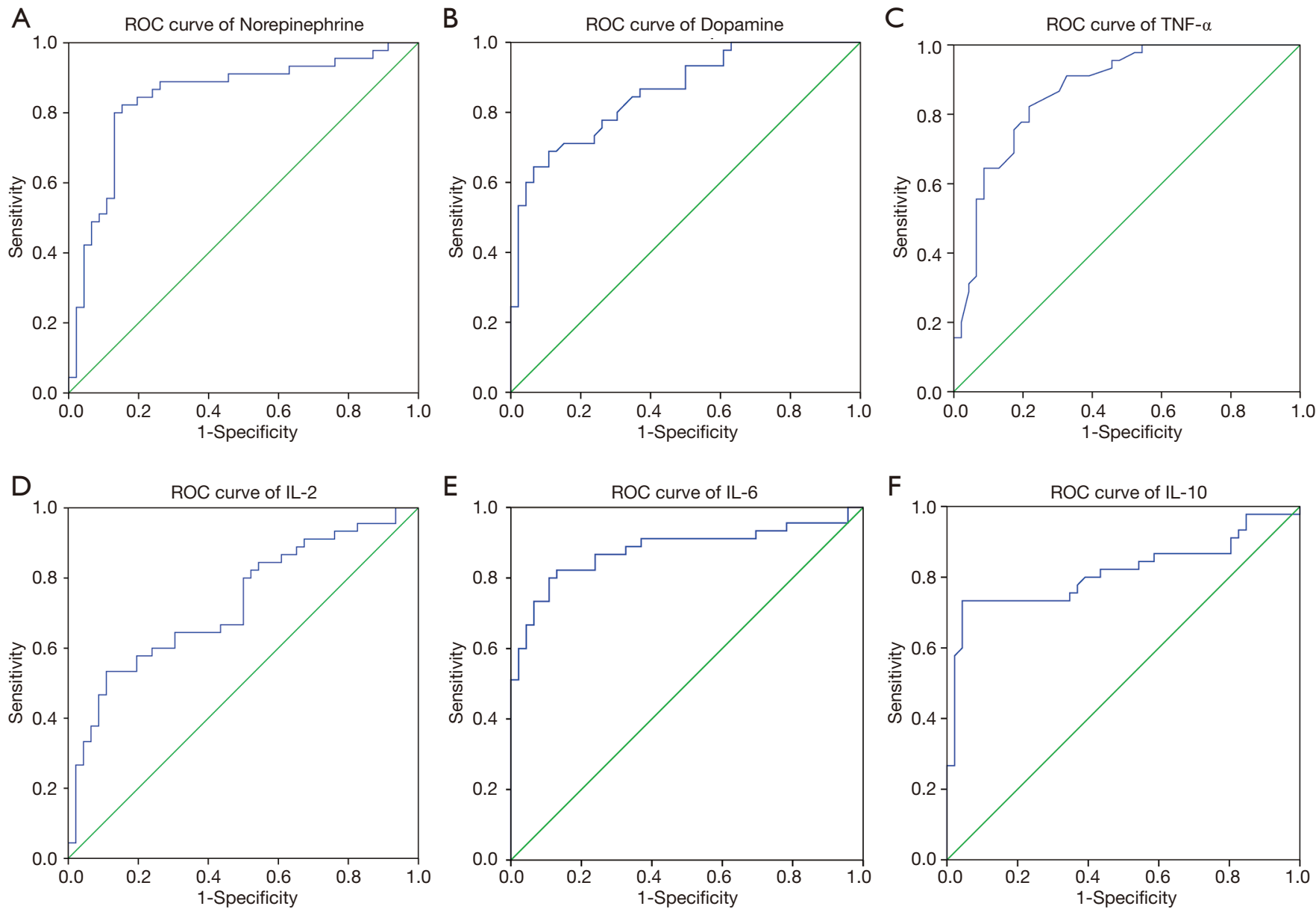

Figure 2 Receiver-operating characteristic (ROC) analysis for operating room nurses with post-traumatic stress disorder. (A) ROC analysis for norepinephrine $(\mathrm{P}<0.05$, area under the ROC curve: 0.845 , sensitivity: $84.8 \%$, and specificity: $82.2 \%)$; (B) ROC analysis for dopamine $(\mathrm{P}<0.05$, area under the ROC curve: 0.727 , sensitivity: $53.3 \%$, and specificity: $89.1 \%)$; (C) ROC analysis for tumor necrosis factor- $\alpha$ (TNF- $\alpha$; $\mathrm{P}<0.05$, area under the ROC curve: 0.859, sensitivity: 68.9\%, and specificity: $89.1 \%$ ); (D) ROC analysis for interleukin (IL)-2 (P<0.05, area under the ROC curve: 0.871, sensitivity: $82.2 \%$, and specificity: $78.3 \%$ ); (E) ROC analysis for IL-6 ( $<<0.05$, area under the ROC curve: 0.879 , sensitivity: $82.2 \%$, and specificity: $87.0 \%$ ); (F) ROC analysis for $\mathrm{IL}-10$ ( $\mathrm{P}<0.05$, area under the ROC curve: 0.814 , sensitivity: $73.3 \%$, and specificity: 95.7\%). Non-PTSD group: $\mathrm{n}=46$; PTSD group: $\mathrm{n}=45$; PTSD, post-traumatic stress disorder.

which may lead to abnormal reaction of nervous system. Cytokines are produced by immune cells and maintain homeostasis by regulating the immune system and other biologic systems, such as the hypothalamic-pituitary adrenal axis (25). Previous studies have indicated that cytokines may result in the pathophysiology of PTSD through a variety of mechanisms, which are independent of comorbid depression and physical diseases (26-28). Recently published studies have demonstrated the innate immune dysregulation in PTSD, and this dysregulation may be a risk factor for the development of this disorder (29-31). In line with findings of the present study, previous studies have demonstrated that PTSD patients have increased levels of IL-2, IL6 , IL-10, and TNF- $\alpha(25,32,33)$. In the present study, we found that plasma monoamine neurotransmitter and serum cytokine levels had a good diagnostic effect on the occurrence of PTSD among operating room nurses, and these could be used to predict PTSD.

Furthermore, we also studied cognitive behavior and resilience among operating room nurses with PTSD. The results showed that there was a negative correlation between plasma monoamine neurotransmitter and serum cytokine 

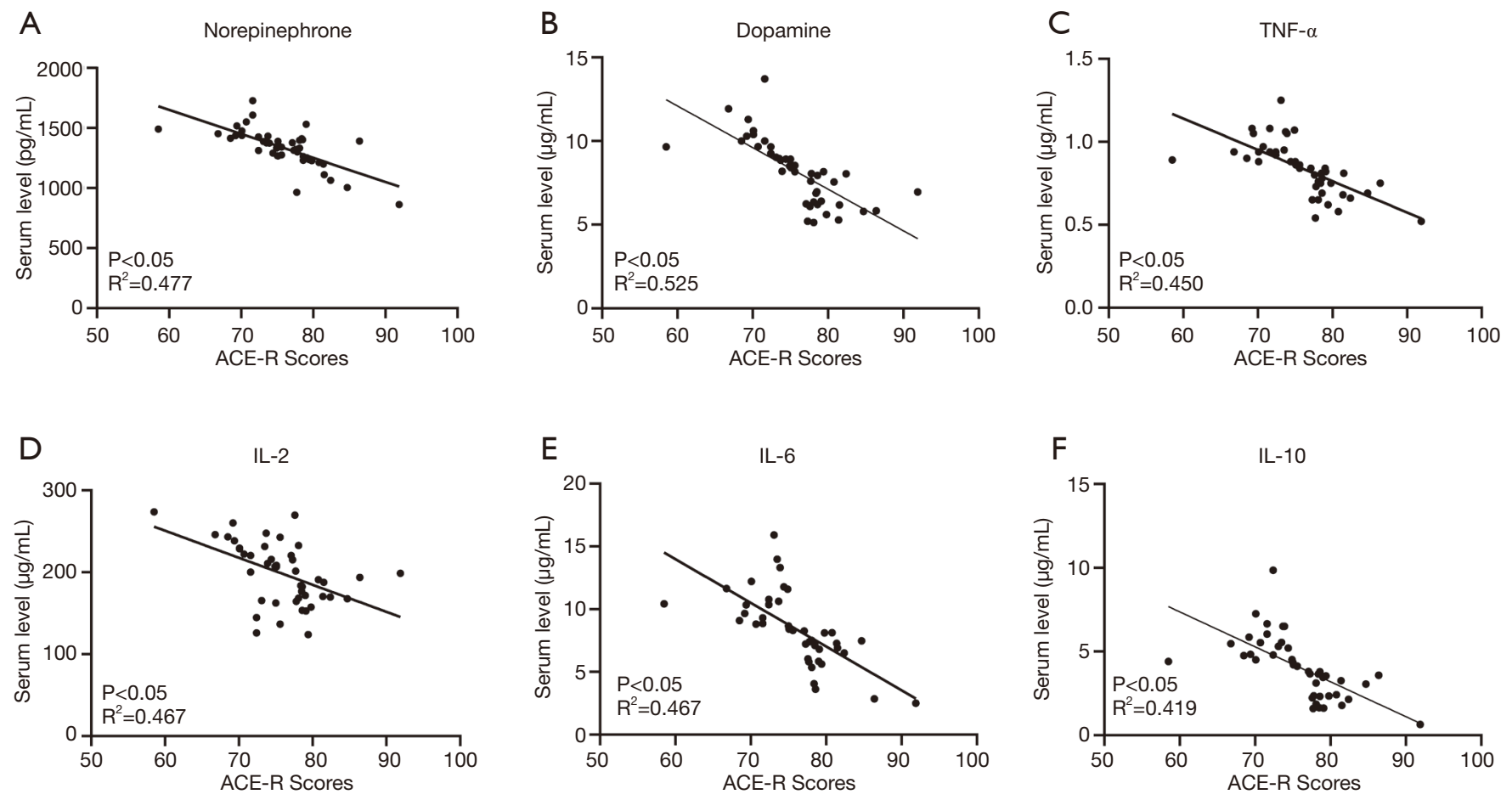

Figure 3 Correlation between monoamine neurotransmitter and cytokine levels and Addenbrooke's Cognitive Examination-Revised (ACE-R) cognition scores. (A) Correlation between norepinephrine and ACE- $\mathrm{R}$ scores $\left(\mathrm{P}<0.05, \mathrm{R}^{2}=0.477\right)$; (B) correlation between dopamine and ACE-R scores $\left(\mathrm{P}<0.05, \mathrm{R}^{2}=0.525\right)$; $(\mathrm{C})$ correlation analysis tumor necrosis factor $-\alpha(T N F-\alpha)$ and $A C E-R$ scores $\left(P<0.05, R^{2}=0.450\right)$; $(D)$ correlation between interleukin (IL)-2 and ACE-R scores $\left(\mathrm{P}<0.05, \mathrm{R}^{2}=0.236\right)$; (E) correlation between IL-6 and ACE-R scores $(\mathrm{P}<0.05$, $\left.\mathrm{R}^{2}=0.467\right)$; $(\mathrm{F})$ correlation between IL-10 and ACE- $\mathrm{R}$ scores $\left(\mathrm{P}<0.05, \mathrm{R}^{2}=0.419\right)$. Pearson's correlation coefficient test was utilized. $\mathrm{n}=45$.

levels and the ACE-R score among operating room nurses with PTSD and with the correlation of resilience. It has been suggested that the higher the neurotransmitter and cytokine levels, the greater the PTSD severity. Individuals exposure to stress during childhood and adolescence are more likely to have neuropsychiatric disorders later in life (34). Rescue and ambulance personnel have been found to have PTSD symptoms for a PTSD diagnosis $(35,36)$. Healthcare workers are usually faced with direct and indirect trauma in the hospital setting; for example, workplace violence, witnessing other people's direct experience of violence in the workplace, caring for dying patients, and threats of severe injury or exposure to trauma (37-39). It has been reported that PTSD patients have different degrees of cognitive impairment (19). One recent study that focused on resilience in functional neurological disorders (FNDs) found that patients with FND had decreased resilience (40).
The findings of the present study show that there is a correlation between monoamine neurotransmitter and cytokine levels and the occurrence of PTSD among operating room nurses. The higher the neurotransmitter and cytokine levels, the greater the PTSD severity. Previous study show that they study the changes of neurotransmitter levels in patients with post-traumatic stress disorder; while our article studies the relationship between monoamine neurotransmitters and immune factors on the occurrence of PTSD and clinical manifestations in operating room nurses. Generally speaking, the research subjects are patients who have experienced traumatic events, but seldom involved medical staff. Our innovation is that the research subjects are operating room nurses who have experienced traumatic events. However, due to the complexity of pathogenesis of PTSD, more relevant research is needed to explore the underlying mechanisms. 

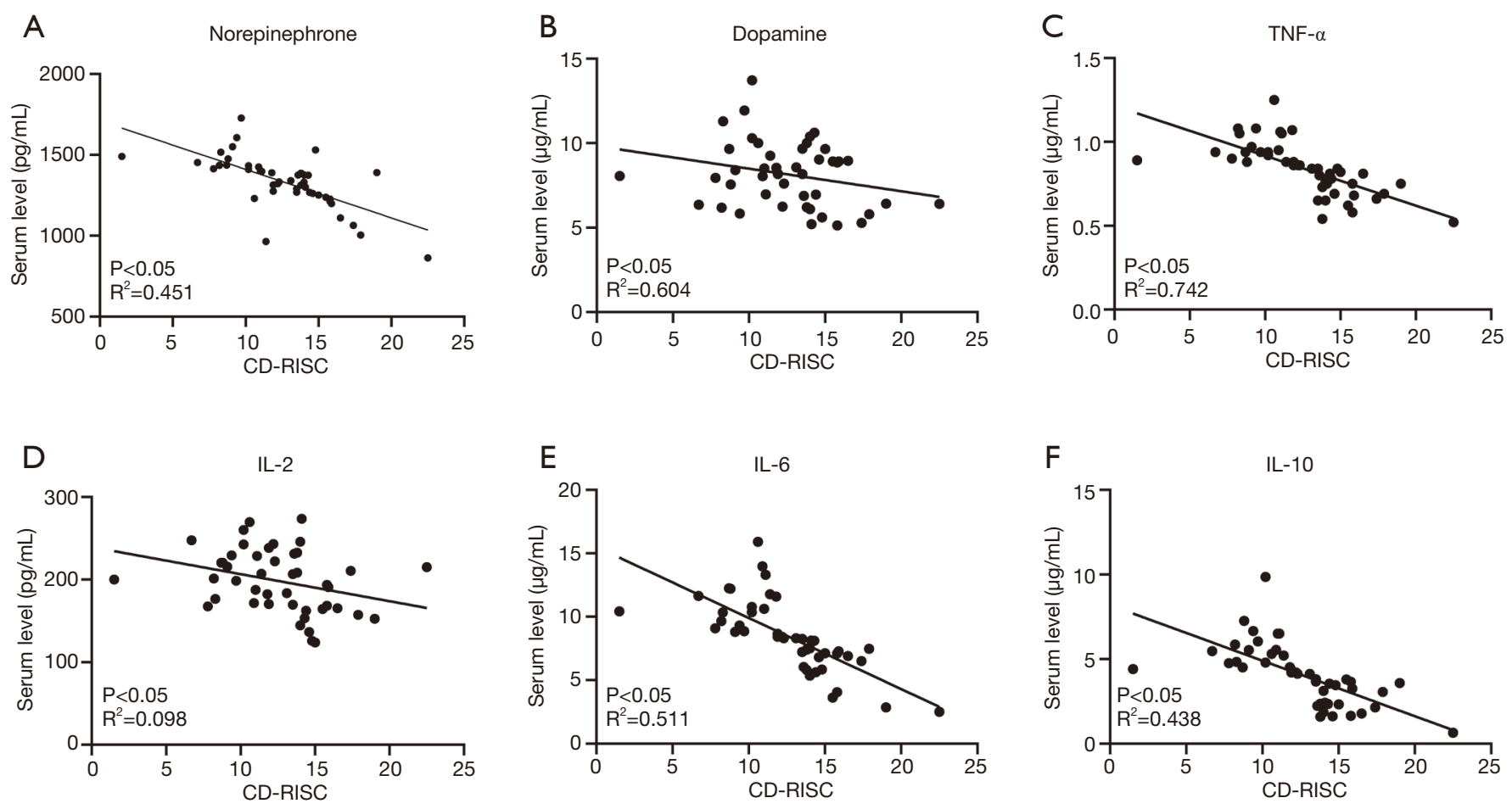

Figure 4 Correlation between monoamine neurotransmitter and cytokine levels and Connor-Davidson Resilience Scale (CD-RISC). (A) Correlation between norepinephrine and CD-RISC $\left(\mathrm{P}<0.05, \mathrm{R}^{2}=0.451\right)$; (B) correlation between dopamine and CD-RISC ( $<<0.05$, $\left.\mathrm{R}^{2}=0.604\right)$; (C) correlation between tumor necrosis factor- $\alpha(\mathrm{TNF}-\alpha)$ and CD-RISC $\left(\mathrm{P}<0.05, \mathrm{R}^{2}=0.472\right)$; (D) correlation between interleukin (IL)-2 and CD-RISC ( $\left.\mathrm{P}<0.05, \mathrm{R}^{2}=0.098\right)$; (E) correlation between IL-6 and CD-RISC $\left(\mathrm{P}<0.05, \mathrm{R}^{2}=0.511\right)$; (F) correlation between IL-10 and CD-RISC $\left(\mathrm{P}<0.05, \mathrm{R}^{2}=0.438\right)$. Pearson's correlation coefficient test was utilized. $\mathrm{n}=45$.

\section{Acknowledgments}

Funding: The present study was supported by the Key Technology Project in Hainan, China (No. ZDYF2019159) and Natural Science Foundation of Hainan Province (818MS161).

\section{Footnote}

Reporting Checklist: The authors have completed the MDAR reporting checklist. Available at http://dx.doi.org/10.21037/ apm-20-1829

Data Sharing Statement: Available at http://dx.doi. org/10.21037/apm-20-1829

Conflicts of Interest: All authors have completed the ICMJE uniform disclosure form (available at http://dx.doi. org/10.21037/apm-20-1829). The authors have no conflicts of interest to declare.
Ethical Statement: The authors are accountable for all aspects of the work in ensuring that questions related to the accuracy or integrity of any part of the work are appropriately investigated and resolved. The present study was approved by the Medical Ethics Committee of the Hainan General Hospital (No.: 20191023), and the participants provided signed informed consent. The study was conducted in accordance with the Declaration of Helsinki (as revised in 2013).

Open Access Statement: This is an Open Access article distributed in accordance with the Creative Commons Attribution-NonCommercial-NoDerivs 4.0 International License (CC BY-NC-ND 4.0), which permits the noncommercial replication and distribution of the article with the strict proviso that no changes or edits are made and the original work is properly cited (including links to both the formal publication through the relevant DOI and the license). See: https://creativecommons.org/licenses/by-nc-nd/4.0/. 


\section{References}

1. Cornelis MC, Nugent NR, Amstadter AB, et al. Genetics of post-traumatic stress disorder: review and recommendations for genome-wide association studies. Curr Psychiatry Rep 2010;12:313-26.

2. Miller MW, Sadeh N. Traumatic stress, oxidative stress and post-traumatic stress disorder: neurodegeneration and the accelerated-aging hypothesis. Mol Psychiatry 2014;19:1156-62.

3. Almli LM, Fani N, Smith AK, et al. Genetic approaches to understanding post-traumatic stress disorder. Int J Neuropsychopharmacol 2014;17:355-70.

4. Hughes KC, Shin LM, Functional neuroimaging studies of post-traumatic stress disorder. Expert Rev Neurother 2011;11:275-85.

5. Rabinak CA, Angstadt M, Welsh RC, et al. Altered amygdala resting-state functional connectivity in posttraumatic stress disorder. Front Psychiatry 2011;2:62.

6. Javidi H, Yadollahie M. Post-traumatic Stress Disorder. Int J Occup Environ Med 2012;3:2-9.

7. Zhang Y, Zhou X, Yang L, et al. Comparative efficacy and acceptability of psychotherapies for post-traumatic stress disorder in children and adolescents: study protocol for a systematic review and network meta-analysis. BMJ Open 2018;8:e020198.

8. Yan J, Kuzhiumparambil U, Bandodkar S, et al. Development and validation of a simple, rapid and sensitive LC-MS/MS method for the measurement of urinary neurotransmitters and their metabolites. Anal Bioanal Chem 2017;409:7191-9.

9. Sharma S, Moon CS, Khogali A, et al. Biomarkers in Parkinson's disease (recent update). Neurochem Int 2013;63:201-29.

10. Zhao XE, He Y, Li M, et al. Analysis of amino acid and monoamine neurotransmitters and their metabolites in rat urine of Alzheimer's disease using in situ ultrasound-assisted derivatization dispersive liquid-liquid microextraction with UHPLC-MS/MS. J Pharm Biomed Anal 2017;135:186-98.

11. Hasanzadeh M, Shadjou N, Omidinia E. A novel electroanalytical method for simultaneous detection of two neurotransmitter dopamine and serotonin in human serum. J Neurosci Methods 2013;219:52-60.

12. Mellon SH, Gautam A, Hammamieh R, et al. Metabolism, Metabolomics, and Inflammation in Posttraumatic Stress Disorder. Biol Psychiatry 2018;83:866-75.

13. Mealer M, Jones J, Posttraumatic stress disorder in the nursing population: a concept analysis. Nurs Forum 2013;48:279-88.

14. Mealer M, Burnham EL, Goode CJ, et al. The prevalence and impact of post traumatic stress disorder and burnout syndrome in nurses. Depress Anxiety 2009;26:1118-26.

15. Czaja AS, Moss M, Mealer M. Symptoms of posttraumatic stress disorder among pediatric acute care nurses. J Pediatr Nurs 2012;27:357-65.

16. Weathers FW, Litz BT, Herman DS, et al. PTSD Checklist: Reliability, validity, and diagnostic utility. January 1993.

17. Rotomskis A, Margeviciute R, Germanavicius A, et al. Differential diagnosis of depression and Alzheimer's disease with the Addenbrooke's Cognitive ExaminationRevised (ACE-R). BMC Neurol 2015;15:57.

18. Campbell-Sills L, Stein MB. Psychometric analysis and refinement of the Connor-davidson Resilience Scale (CDRISC): Validation of a 10-item measure of resilience. J Trauma Stress 2007;20:1019-28.

19. Twamley EW, Allard CB, Thorp SR, et al. Cognitive impairment and functioning in PTSD related to intimate partner violence. J Int Neuropsychol Soc 2009;15:879-87.

20. Cho GJ, Kang J. Type D personality and post-traumatic stress disorder symptoms among intensive care unit nurses: The mediating effect of resilience. PLoS One 2017;12:e0175067.

21. Brunello N, Davidson JR, Deahl M, et al. Posttraumatic stress disorder: diagnosis and epidemiology, comorbidity and social consequences, biology and treatment. Neuropsychobiology 2001;43:150-62.

22. Shin LM, Rauch SL, Pitman RK. Amygdala, medial prefrontal cortex, and hippocampal function in PTSD. Ann N Y Acad Sci 2006;1071:67-79.

23. Milad MR, Quirk GJ, Neurons in medial prefrontal cortex signal memory for fear extinction. Nature 2002;420:70-4.

24. Bailey CR, Cordell E, Sobin SM, et al. Recent progress in understanding the pathophysiology of post-traumatic stress disorder: implications for targeted pharmacological treatment. CNS Drugs 2013;27:221-32.

25. Wong CM. Post-traumatic stress disorder: advances in psychoneuroimmunology. Psychiatr Clin North Am 2002;25:369-383, vii.

26. Plantinga L, Bremner JD, Miller AH, et al. Association between posttraumatic stress disorder and inflammation: a twin study. Brain Behav Immun 2013;30:125-32.

27. Tursich M, Neufeld RW, Frewen PA, et al. Association of trauma exposure with proinflammatory activity: a transdiagnostic meta-analysis. Transl Psychiatry 
2014;4:e413.

28. Lindqvist D, Wolkowitz OM, Mellon S, et al. Proinflammatory milieu in combat-related PTSD is independent of depression and early life stress. Brain Behav Immun 2014;42:81-8.

29. Guardado P, Olivera A, Rusch HL, et al. Altered gene expression of the innate immune, neuroendocrine, and nuclear factor-kappa B (NF-kappaB) systems is associated with posttraumatic stress disorder in military personnel. J Anxiety Disord 2016;38:9-20.

30. Tylee DS, Chandler SD, Nievergelt CM, et al. Bloodbased gene-expression biomarkers of post-traumatic stress disorder among deployed marines: A pilot study. Psychoneuroendocrinology 2015;51:472-94.

31. Breen MS, Maihofer AX, Glatt SJ, et al. Gene networks specific for innate immunity define post-traumatic stress disorder. Mol Psychiatry 2015;20:1538-45.

32. Chen T, Guo M, Gao Y, et al. A comparative study on the levels of serum cytokines and cortisol among posttraumatic stress disorder patients of $\mathrm{Li}$ and Han ethnicities in Hainan. Chin Med J (Engl) 2014;127:2771-4.

33. Lerman I, Davis BA, Bertram TM, et al. Posttraumatic stress disorder influences the nociceptive and intrathecal cytokine response to a painful stimulus in combat veterans. Psychoneuroendocrinology 2016;73:99-108.

34. McCrory E, De Brito SA, Viding E. The link between child abuse and psychopathology: a review

Cite this article as: Ke Y, Zhu H, Huang Y, Cheng L, Wu X, Chen N. Correlation between monoamine neurotransmitter and cytokine levels and the occurrence of post-traumatic stress disorder among operating room nurses. Ann Palliat Med 2020;9(6):3947-3956. doi: 10.21037/apm-20-1829 of neurobiological and genetic research. J R Soc Med 2012;105:151-6.

35. Alexander DA, Klein S. Ambulance personnel and critical incidents: impact of accident and emergency work on mental health and emotional well-being. Br J Psychiatry 2001;178:76-81.

36. Jonsson A, Segesten K, Mattsson B. Post-traumatic stress among Swedish ambulance personnel. Emerg Med J 2003;20:79-84.

37. Shi L, Wang L, Jia X, et al. Prevalence and correlates of symptoms of post-traumatic stress disorder among Chinese healthcare workers exposed to physical violence: a cross-sectional study. BMJ Open 2017;7:e016810.

38. Laposa JM, Alden LE, Fullerton LM. Work stress and posttraumatic stress disorder in ED nurses/personnel. J Emerg Nurs 2003;29:23-8.

39. de Boer J, Lok A, Van't Verlaat E, et al. Work-related critical incidents in hospital-based health care providers and the risk of post-traumatic stress symptoms, anxiety, and depression: a meta-analysis. Soc Sci Med 2011;73:316-26.

40. Jalilianhasanpour R, Williams B, Gilman I, et al. Resilience linked to personality dimensions, alexithymia and affective symptoms in motor functional neurological disorders. J Psychosom Res 2018;107:55-61.

(English Language Editor: R. Scott) 